\title{
THC:CBD Spray and MS Spasticity Symptoms: Data from Latest Studies
}

\author{
Tiina Rekand \\ Bergen Hospital and University of Bergen, Bergen, Norway
}

\author{
Key Words \\ THC C CBD · Cannabinoids · Multiple sclerosis · Spasticity · \\ Cognition $\cdot$ Driving $\cdot$ Registry
}

\begin{abstract}
New clinical experience with 9-delta-tetrahydocannabinol (THC) and cannabidiol (CBD) oromucosal spray (Sativex ${ }^{\circledR}$ ) involving more than an additional 1,000 patients with MS spasticity (approximately 150 in clinical studies and 900 in postmarketing surveillance studies) have become available in 2013 and are reviewed. A randomized, placebo controlled long-term follow-up clinical trial with THC:CBD spray versus placebo demonstrated that it was not associated with cognitive decline, depression or significant mood changes after 12 months of treatment. Furthermore, in a prospective observational pilot study involving 33 patients ( $60 \%$ female) aged 33-68 years and a mean disease duration of 6.6 years, THC:CBD oromucosal spray did not adversely influence standard driving ability in patients with moderate to severe MS spasticity. Other new long term observational data about the use of THC:CBD oromucosal spray in clinical practice are available from patient registries in the UK, Germany and Spain. Findings to date reinforce the efficacy and safety observed in Phase III clinical trials. It is of interest that in practice average dosages used by patients tended to be lower than those reported in clinical studies (5-6.4 vs. $>8$ sprays/day),
\end{abstract}

and effectiveness was maintained in the majority of patients. Importantly, no additional safety concerns were identified in the registry studies which included findings from patients who have been treated for prolonged periods (in the German/UK registry $45 \%$ of patients had $>2$ years exposure). Thus, these new data support a positive benefit-risk relationship for THC:CBD oromucosal spray during longer-term use.

(c) 2014 S. Karger AG, Basel

\section{Introduction}

Sativex ${ }^{\circledR}$ is a unique cannabinoid-based medicine comprising a 1:1 mixture of 9-delta-tetrahydocannabinol (THC) and cannabidiol (CBD). THC:CBD oromucosal spray is now available in many countries as an add-on treatment for adult MS patients with moderate to severe spasticity who do not adequately respond to first-line antispasticity therapy.

With regards the clinical assessment of THC:CBD oromucosal spray, published randomized and long-term clinical trials have been favorable in terms efficacy and safety findings [1-5]. For example a 16-week pivotal phase III placebo-controlled trial reported by Novotna and colleagues provided both interesting and convincing findings [1]. This study employed an enriched design and was split into 2 phases which reflect the approved clinical

\section{KARGER}

E-Mail karger@karger.com

www.karger.com/ene
C 2014 S. Karger AG, Basel

0014-3022/14/0717-0004\$39.50/0
Tiina Rekand

Bergen Hospital and University of Bergen

Jonas Lies vei 65

NO-5021 Bergen (Norway)

E-Mail tiina.rekand@ @else-bergen.no 
use. Only those participants who showed an initial response to THC:CBD oromucosal spray during the preliminary 4-week single-blind phase (phase A), defined as $a \geq 20 \%$ reduction in the spasticity $0-10$ numerical rating scale (NRS) score, were eligible for randomisation in the subsequent 12-week double-blind phase (phase B) of the study. Of 572 patients who entered phase A across 51 study sites in Europe, 241 were initial responders and met the criteria to progress to phase B. After 12 weeks' treatment, the intention-to-treat analysis showed a significant difference in favour of THC:CBD oromucosal spray over placebo $(\mathrm{p}=0.0002)$. THC:CBD oromucosal spray was also significantly superior to placebo for reductions in spasm frequency $(\mathrm{p}=0.005)$ and sleep disruption $(\mathrm{p}<$ 0.0001), and improvements in Barthel Activities of Daily Living ( $\mathrm{p}=0.0067)$, Physician Global Impression of Change ( $p=0.005)$, Subject Global Impression of Change $(\mathrm{p}=0.023)$ and Carer Global Impression of Change in Function ( $p=0.005)$, all of which were secondary endpoints. This study confirmed the positive findings from a number of earlier clinical trials with THC:CBD oromucosal spray in patients with MS spasticity [2-5].

Clinical experience, both in terms of efficacy and safety, with THC:CBD oromucosal spray in patients with MS-related spasticity has been accumulating since the first reported studies in 2003. Findings from the Sativex Clinical Program involved more than 1,500 MS patients and a cumulative of $>1,500$ patient-years of therapeutic usage. This included $>800$ patients who had been treated continuously for 6 months or more in controlled studies $[6,7]$. In total there were 5 Phase III randomized clinical trials supporting the clinical development [1-5].

The aim of this article will be to provide an update of new clinical experience with THC:CBD oromucosal spray involving an additional 1,000 patients with MS spasticity (approximately 150 in clinical studies investigating the effects of the drug on driving ability (observational) and cognitive function (randomized clinical trial), and 900 in post-marketing surveillance studies). In total there are now $>20,000$ patient-years of clinical experience with THC:CBD oromucosal spray.

\section{Effects of THC:CBD Oromucosal Spray on Driving Ability}

Because MS symptoms such as movement, cognition or attention impairment and spasms can cause problems with driving, it is important that MS medications do not impose additional restrictions on patients who are still able to drive. Given the nature of the active ingredients in THC:CBD oromucosal spray some health authorities (e.g. Germany and Denmark) expressed interest about the possible effects of the medication on users' driving abilities and this formed the basis of a study recently presented by Friedel and colleagues [8]. The purpose of this prospective observational pilot study was to explore whetherTHC:CBD oromucosal spray might impair the driving ability of MS patients who were starting treatment under approved and everyday conditions in three MS specialist centres in Germany. A battery of validated tests was used to assess driving ability in still-driving adult MS patients starting treatment with THC:CBD oromucosal spray for moderate to severe resistant MS-related spasticity. The computer tests covered 5 specific driving-related ability dimensions:

- Visual orientation (Visual Pursuit Test)

- Attention and concentration (Concentration Cognitrone Test)

- Reactive stress tolerance (Stress Tolerance Determination Test)

- Attention and reaction speed (Reaction Speed: Motor Speed Reaction)

- Observational ability and skill in gaining an overview (Adaptive Tachistoscopic Traffic Perception Test).

Evaluations were performed at baseline, before the new medication was started (study enrolment) and after 4-6 weeks' treatment with THC:CBD oromucosal spray (final visit).

33 patients (60\% female) aged 33-68 (mean 48) years and a mean disease duration of 6.6 years were enrolled. The decision to start treatment with THC:CBD oromucosal spray was taken prior to study enrolment and all patients were driving (at least once weekly) at the time of enrolment. During the course of the study, THC:CBD oromucosal spray was used in conjunction with other medications, mainly tolperisone $(n=8)$ or baclofen $(n=$ 5). Two patients discontinued treatment prior to final visit because of lack of efficacy $(n=1)$ or lack of tolerability $(\mathrm{n}=1)$. The mean dose of THC:CBD oromucosal spray at final visit in the remaining 31 patients was 5 sprays/day.

At the final visit, the overall mean score for 5 driving tests improved by $+2.1 \%$ (NS). There were no statistically significant changes versus baseline in mean scores for 4 of the 5 driving tests (fig. 1): Visual Pursuit Test $-1.5 \%$; Concentration Cognitrone Test $+1.2 \%$; Reaction Speed: Motor Speed Reaction $+1.6 \%$; Adaptive Tachistoscopic Traffic Perception Test $+3.0 \%$. A statistically significant improvement versus baseline in favor of THC:CBD oromucosal spray was recorded for the Stress Tolerance Determination Test $(+6.1 \%$; $\mathrm{p}=0.0255)$ (fig. 1$)$. 
Fig. 1. Effect of THC:CBD oromucosal spray on 5 specific driving-related ability dimensions in patients with moderate to severe resistant MS-related spasticity. ${ }^{*} \mathrm{p}=$ 0.0255 versus baseline.

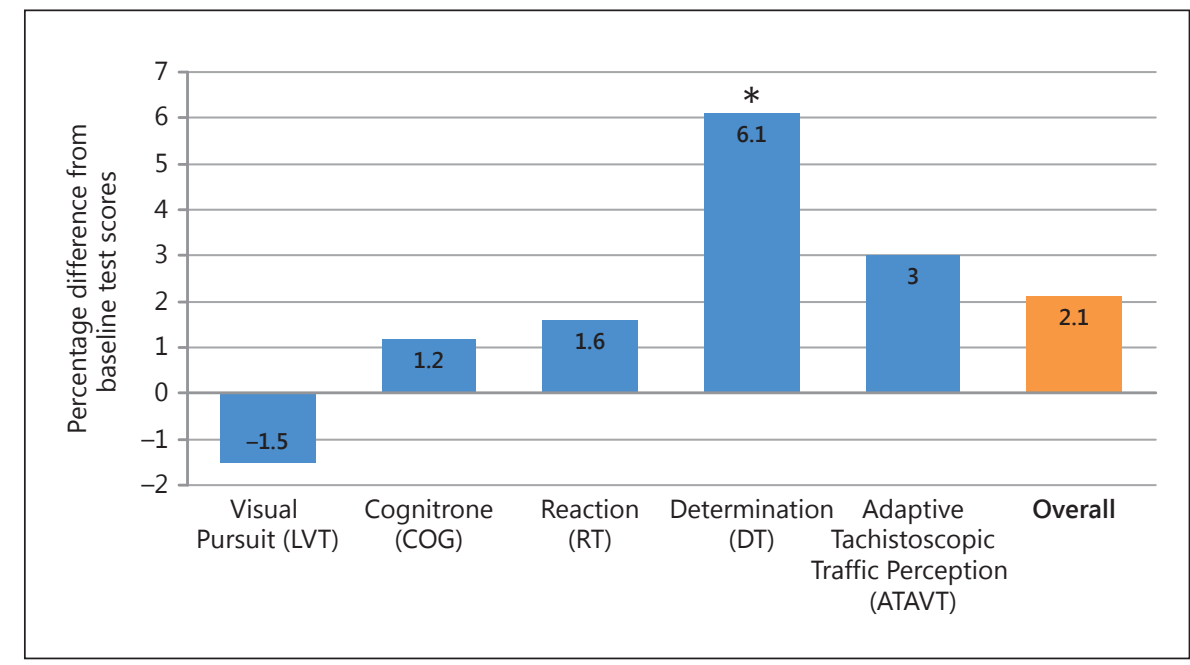

Findings relating to the efficacy and safety of THC:CBD:

- The mean spasticity 0-10 NRS score decreased from $6.0( \pm 1.76)$ at baseline to $3.6( \pm 1.73)$ at final visit $(\mathrm{p}<$ 0.0001 ) (fig. 1).

- At baseline, 3, 23 and 7 patients, respectively, rated their spasticity severity as mild, moderate or severe; corresponding values at final visit were 9, 23 and 1, respectively.

- In patients reporting spasms $(n=20)$, the mean number of spasms/day decreased from 38.3 (range: 1-100) at baseline to 17.4 (range: 1-60) at final visit.

- A total of 5 non-serious adverse events were reported in 4 patients: dizziness ( 2 events), ligament sprain (1), thrombosis (1) and vertigo (1). Only dizziness and vertigo were considered treatment related. All adverse events were rated as mild or moderate in intensity.

Thus, based upon the results of this prospective observational pilot study THC:CBD oromucosal spray did not adversely influence standard driving ability tests in patients with moderate to severe MS spasticity. Furthermore, over the course of the study, it improved MS spasticity, reduced the number of spasms and was generally well tolerated.

\section{Effects of THC:CBD Oromucosal Spray on Cognitive Function and Mood}

During the approval of THC:CBD oromucosal spray for MS spasticity its long-term impact on cognitive function and mood was not thoroughly evaluated. As part of the usual risk-management plan for any new approved medication, agreed between European regulatory agencies, a clinical trial was designed to investigate this concern and included patients with moderate to severe spasticity, despite treatment with other anti-spasticity agents, who were randomised to receive THC:CBD or matching placebo for 12 months. The primary endpoint was the change in the Paced Auditory Serial Addition Test 2 and 3 (PASAT) from baseline to end of study. Secondary endpoints included the Beck Depression Inventory-II (BDIII), the subject, physician and caregiver global impression of change (GIC), and the Columbia Suicidality Severity Rating Scale.

121 subjects ( $63 \%$ females) of mean \pm SD (standard deviation) age $49 \pm 9.6$ years and mean duration of MS $13.9 \pm 8.6$ years were randomised to active or placebo treatment. Mean dose at the final visit was 6.4 sprays/day in the active treatment group, with an exposure duration of 294 days, compared with 300 days in the placebo group. Concomitant anti-spasticity medication included baclofen (54\% of sample), tizanidine (18\%), benzodiazepines (30\%), and/or gabapentinoids (18\%). $49 \%$ of subjects had relapsing-remitting MS, and $39 \%$ secondary progressive MS. Baseline PASAT score in the THC:CBD group was 59.4 and in the placebo group was 62.1 (fig. 2). After 12 months, the mean change was +6.8 in both groups. The change in the BDI-II score was -3.1 in the active group compared with -2.4 in the placebo group (fig. 2). Neither difference approached statistical significance. Patient, physician and caregiver GIC were all significantly in favor of THC:CBD ( $<<0.0001, \mathrm{p}=0.0014$, and $\mathrm{p}=0.0042$, respectively) (fig. 3 ). 


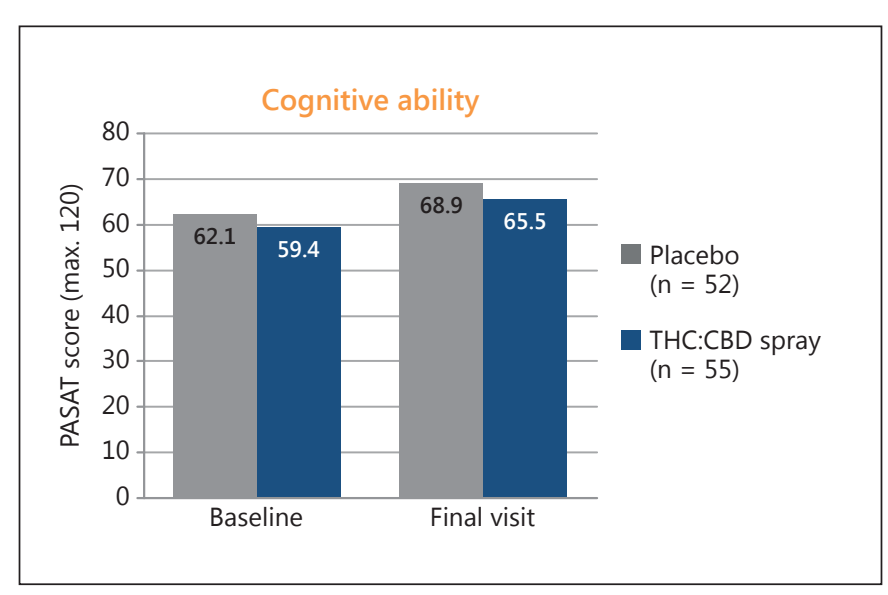

Fig. 2. Effects of THC:CBD oromucosal spray and placebo on cognitive ability of MS patients with spasticity treated for 50 weeks.

Table 1. Tolerability of THC:CBD oromucosal spray and placebo in MS patients with spasticity treated for 50 weeks

\begin{tabular}{lcc}
\hline & $\begin{array}{l}\text { THC:CBD spray } \\
(\mathrm{n}=62)\end{array}$ & $\begin{array}{c}\text { Placebo } \\
(\mathrm{n}=59)\end{array}$ \\
\hline Any AE & $39(63 \%)$ & $19(32 \%)$ \\
Treatment-related & $25(40.3 \%)$ & $5(8.5 \%)$ \\
Vertigo & $6(9.7 \%)$ & 0 \\
Dizziness & $5(8.1 \%)$ & 0 \\
Fatigue & $5(8.1 \%)$ & $1(1.7 \%)$ \\
Muscle spasticity & $5(8.1 \%)$ & $2(3.4 \%)$ \\
Respiratory tract infection & 0 & $3(5 \%)$ \\
Withdrawals from study & $12(19 \%)$ & $11(19 \%)$ \\
AE & 8 & 2 \\
Withdrew consent & 4 & 7 \\
Lost to follow-up & 0 & 1 \\
Investigator decision & 0 & 1 \\
\hline
\end{tabular}

Withdrawal from treatment occurred in $19 \%$ of subjects in both the THC:CBD and placebo groups (table 1). Overall, adverse events (AEs) were reported by $63 \%$ of subjects on THC:CBD and $32 \%$ of subjects on placebo. AEs led to cessation of study medication in 8 patients on THC:CBD and two on placebo. One of 5 reported serious AEs was considered related to THC:CBD. There was one case of suicidal ideation, in a subject taking placebo. No psychotic disorders were reported.

In summary, long-term treatment with THC:CBD spray was not associated with cognitive decline, depression or significant changes in mood. Efficacy, assessed

THC:CBD Spray and MS Spasticity Symptoms

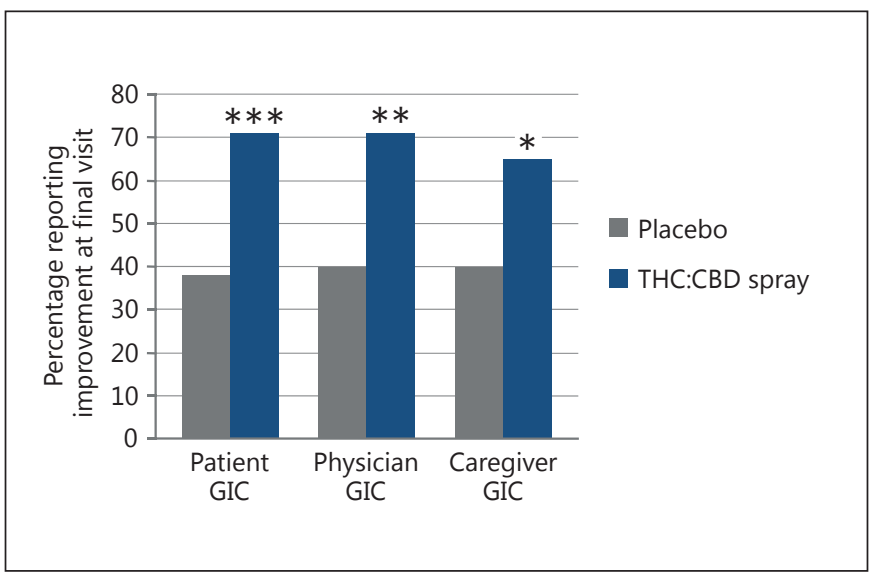

Fig. 3. Effects of THC:CBD oromucosal spray and placebo on patient, physician and caregiver Global Impression of Change (GIC) in MS patients with spasticity treated for 50 weeks. ${ }^{*} \mathrm{p}=$ $0.0042 ;{ }^{* *} \mathrm{p}=0.0014 ;{ }^{* * *} \mathrm{p}<0.001$.

separately by patients, physicians and caregivers, was maintained during long-term treatment, and the spray was well-tolerated with no new tolerability issues and few withdrawals.

\section{Post-Marketing Surveillance Registries}

Patient registries have been established in the UK, Germany and Spain to monitor long-term safety outcomes with THC:CBD oromucosal spray in everyday clinical practice. The main objective of these elements in the risk management plan is to identify possible new risks with THC:CBD, quantifying them together with the known tolerability profile associated with the oromucosal spray, by systematically collecting all suspected adverse reactions that occur in patients. Although there are some inter-country differences in the collection methodology (UK/Germany: voluntary retrospective reporting; Spain: prospective cohort follow-up), equivalent key data are being collected in all countries. Focus is on a number of effects which were considered to be of special interest:

- Clinically significant adverse events (AEs)

- Falls

- Suicidal thoughts or attempts

- Significant psychiatric/psychotic events (e.g. mood changes, memory impairment, and confusion/disorientation)

- Changes in driving ability

- Abuse/misuse. 
Table 2. Safety findings of special interest from the UK/German registry $(n=687)$

\begin{tabular}{ll}
\hline & Incidence $(\mathrm{n}=687)$ \\
\hline Any AE, \% (n) & $27.7(190)$ \\
& $10.5(72)$ treatment-related \\
Falls & $4.9(34)$ \\
Depression & $3.3(23)$ \\
Multiple sclerosis & $1.9(13)$ \\
Dizziness & $1.9(13)$ \\
Urinary tract infection & $1.5(10)$ \\
SAEs, \% (n) & $11.5(79)$ \\
& $2.3(16)$ treatment-related \\
\hline
\end{tabular}

Treating physicians rated $84 \%$ of patients as gaining worthwhile benefit from THC:CBD spray.

Table 3. Safety findings of special interest from the Spanish registry

\begin{tabular}{lc}
\hline Tolerability & Incidence $(\mathrm{n}=196)$ \\
\hline Any AE, \% (n) & $19.9 \%(39)$ \\
Significant psychiatric or psychotic event & $2.4 \%(5)$ \\
Reduced driving ability & $0.5 \%(1)$ \\
Fall requiring medical attention & $0 \%(0)$ \\
Suicidal thoughts/attempted suicide & $0 \%(0)$ \\
Abuse/misuse & $0 \%(0)$ \\
Other & $16.8 \%(33)$ \\
\hline
\end{tabular}

More than two-thirds of patients reported deriving benefit from THC:CBD spray, despite having spasticity resistant to treatment with current oral antispasticity agents.

To date almost 900 patients have been enrolled. In the UK/German registry, case report forms for 687 patients have been completed since June 2010 and this analysed sample comprises $57 \%$ females, aged $18-85$ (mean 52 ) years, treated for a median of 570 days (representing $>1,000$ patient-years of exposure) with a mean dose of 5.3 sprays/day ( $45 \%$ had $>2$ years exposure) and $74 \%$ were continuing with treatment. A summary of the latest results from this registry were recently presented by Eltayb and colleagues at ECTRIMS 2013 (table 2) [10].

The main findings to date include:

- Patients tended to use lower doses of THC:CBD oromucosal spray (mean 5.3 sprays/day) in clinical practice compared with controlled clinical studies $(>8$ sprays/day)

- No new safety signals were identified and the rate of significant adverse events was low
- No evidence of addiction, abuse, misuse or impairment of memory was identified

- No apparent loss of driving ability was observed. 207 patients with MS spasticity treated with THC:CBD oromucosal spray have been entered into the Spanish prospective registry since July 2011. Of these, 196 have reached the 6 months interim analysis time-point and 112 the 1-year interim analysis time-point at the time of this data presentation. At 6 months $69 \%$ of the initial patients were continuing with THC:CBD oromucosal spray and the equivalent number was $66 \%$ at 1 -year. The average dosage at both time-points was 6.3 sprays/day. A summary of the latest results from this registry in terms of AEs considered to be of special interest are shown in table 3 .

Thus, the findings from the Spanish registry are in agreement with that of the UK/German registry with no evidence of addiction, abuse or misuse, and no new safety signals. The majority of patients reported treatment benefit. The final results from the Spanish registry analysis are due to be completed in early 2014 .

\section{Conclusions}

In general, the new observational data and experience with using THC:CBD oromucosal spray in everyday clinical practice reinforce the efficacy and safety observed in the regulatory dossier of Phase III clinical trials. It is of interest that in practice the dosages used by patients tended to be lower, on average, than in clinical studies (5-6.4 vs. $>8$ sprays/day), and effectiveness was maintained long term in the majority of patients. Importantly, no additional safety concerns were identified during longer term usage in clinical practice. With regards two specific areas of interest to the regulatory authorities, THC:CBD oromucosal spray did not affect driving ability and it did not have any significant detrimental effects on cognition or mood.

Overall, the data support a positive benefit-risk relationship for THC:CBD oromucosal spray in long-term use.

\section{Disclosure Statement}

T. Rekand received an honorarium from Laboratorios Almirall, SA, for her participation in the Symposium and producing her article, which is included in this Supplement. She has no other relevant affiliations or financial involvement with any organization or entity with a financial interest in or financial conflict with the subject matter or materials discussed in the manuscript apart from those disclosed. Writing assistance was provided by Content Ed Net, with funding from Laboratorios Almirall, SA. 


\section{References}

1 Novotna A, Mares J, Ratcliffe S, et al: A randomized, double-blind, placebo-controlled, parallel-group, enriched-design study of nabiximols* (Sativex ${ }^{(\circledR)}$ ), as add-on therapy, in subjects with refractory spasticity caused by multiple sclerosis. Eur J Neurol 2011;18: 1122-1131.

$>2$ Collin C, Davies P, Mutiboko IK, Ratcliffe S; for the Sativex Spasticity in MS Study Group: Randomized controlled trial of cannabisbased medicine in spasticity caused by multiple sclerosis. Eur J Neurol 2007;14:290-296.

-3 Collin C, Ehler E, Waberzinek G, Alsindi Z, Davies P, Powell K, et al: A double-blind, randomized, placebo-controlled, parallel-group study of Sativex, in subjects with symptoms of spasticity due to multiple sclerosis. Neurol Res 2010;32:451-459.
4 Wade D, Makela P, Robson P, House H, Bateman C: Do cannabis-based medicinal extracts have general or specific effects on symptoms in multiple sclerosis? A double-blind, randomized, placebo-controlled study on $160 \mathrm{pa}-$ tients. Mult Scler 2004;10:434-441.

5 Wade DT, Makela PM, House H, Bateman C, Robson P: Long-term use of a cannabis-based medicine in the treatment of spasticity and other symptoms in multiple sclerosis. Mult Scler 2006;12:639-645.

6 García-Merino A: Endocannabinoid system modulator use in everyday clinical practice in the UK and Spain. Expert Rev Neurother 2013;13:9-13.
7 Robson P: Abuse potential and psychoactive effects of $\delta$-9-tetrahydrocannabinol and cannabidiol oromucosal spray (Sativex), a new cannabinoid medicine. Expert Opin Drug Saf 2011;10:675-685.

8 Friedel M, Tiel-Wilck K, Schreiber H, Lang M: Treatment of resistant MS spasticity with THC:CBD spray and effects on driving ability. Presented at ECTRIMS 2013.

9 Wright S, Vachova MM, Novakova I: The effect of long-term treatment with a prescription cannabis-based THC:CBD oromucosal spray on cognitive function and mood: a 12 month double blind placebo-controlled study in people with spasticity due to Multiple Sclerosis. Presented at ECTRIMS 2013.

10 Eltayb A, Etges T, Wright S: An observational post approval registry study of patients prescribed Sativex ${ }^{\circledR}$. Results from clinical practice. Presented at ECTRIMS 2013. 